\title{
Digital Multi-Probe Temperature Monitoring System for Long-Term on Field Measurements ${ }^{\dagger}$
}

\author{
Leonardo Pantoli *, Mirco Muttillo, Tullio De Rubeis, Iole Nardi, Vincenzo Stornelli and \\ Giuseppe Ferri \\ Department of Industrial and Information Engineering and Economics, University of L'Aquila, 67100 \\ L'Aquila, Italy; mirco.muttillo@graduate.univaq.it (M.W.); tullio.derubeis@graduate.univaq.it (T.D.R.); \\ iole.nardi@graduate.univaq.it (I.N.); vincenzo.stornelli@univaq.it (V.S.); giuseppe.ferri@univaq.it (G.F.) \\ * Correspondence: leonardo.pantoli@univaq.it; Tel.: +39-0862-434444 \\ + Presented at the Eurosensors 2017 Conference, Paris, France, 3-6 September 2017.
}

Published: 11 August 2017

\begin{abstract}
In this work, we present a flexible and multi-probe temperature measurement system, suitable for real time monitoring and long-term applications. The proposed sensor system allows to investigate the thermal performance of different environments such as building areas, walls or external places. The equipment has been characterized both at device and system levels and validated through experimental tests through a comparison with different sensors. The conceived system consists of a control unit with a microcontroller, a user interface and an internal recording section for data storage and management, and digital temperature sensors with a 12-bit resolution, whose number can be freely defined, having the system a modular architecture. The low-cost and open-source characteristics, beyond of its flexibility and capability to manage several sensors placed also very far from the control unit (up to $500 \mathrm{~m}$ ), make the proposed solution a suitable candidate to be used for practical applications that require on-field measurements.
\end{abstract}

Keywords: differential capacitance sensors; bridge-based circuits; CMOS technology; sensor interfaces

\section{Introduction}

In the last years, the use of sensing systems is rapidly increasing and find application in many fields of human activity, from industry to safety and home automation [1-6]. Research is currently moving towards both the development of new materials and also the definition of innovative hardware solutions, often conceived as embedded systems. In this scenario, measurement systems that allow to monitor and describe the thermal behaviour of any object on both wide spatial and temporal scale is something missing in the literature and of great interest in many applied researches, as for instance in the field of construction to characterize the thermal behaviour of buildings in terms of energy efficiency. The solution here presented would fill this gap, proposing an easy-to-use, economic and flexible sensor system that provides a long-term automatic monitoring capability.

\section{The Proposed System}

The system has been designed with a unique data logger able to manage a large number of sensors that can be directly plugged with a wired connection and placed up to $500 \mathrm{~m}$ far from the control unit. The digital probes (DS18B20 provided by Maxim Integrated) have a 12-bit resolution (equivalent to $0.0625^{\circ} \mathrm{C}$ ), are univocally identified through a 64-bit code and communicate with the Unique 1-Wire protocol. The control unit (see Figure 1a,b) embeds a microcontroller programmed accordingly with an optimized algorithm able to automatically recognize and identify the sensors really in use for the considered application. The designed firmware has a pipeline architecture and 
the capability to manage up to 10 different channels and 10 sensors for each channel, resulting in 100 probes.

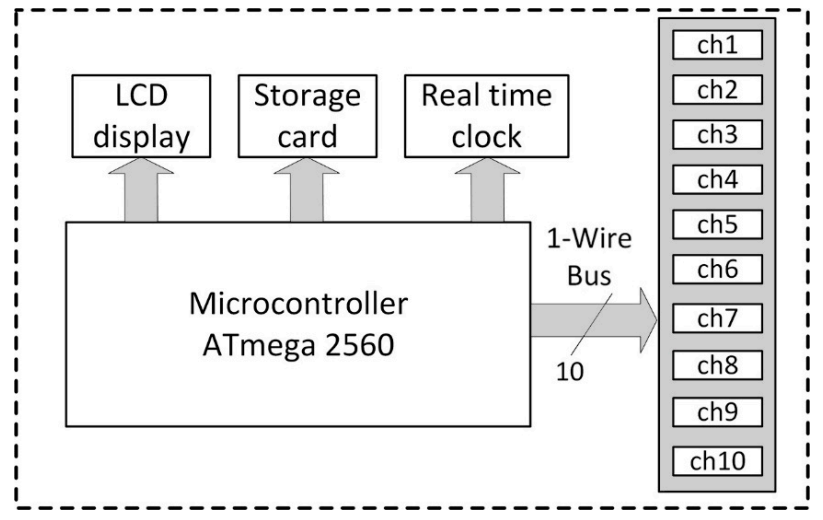

(a)

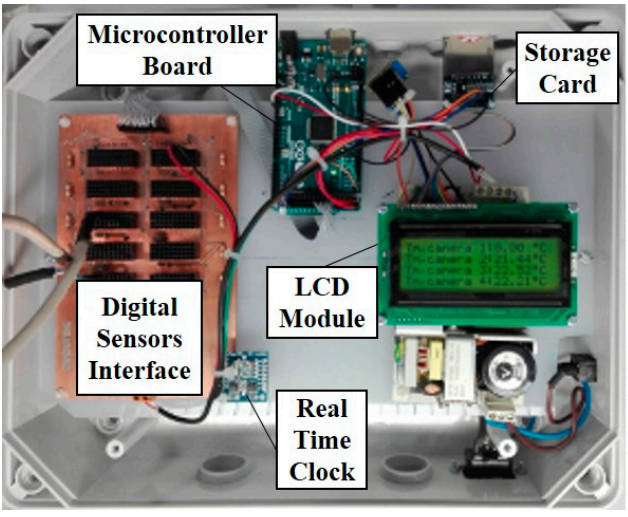

(b)

Figure 1. (a) Control unit architecture; (b) Prototype control unit system box.

The data sensing timing is able to provide the full capability of the system in $1.8 \mathrm{~s}$; the acquisition time of each channel is $900 \mathrm{~ms}$ and all probes connected to the same channel are simultaneously read (Figure 2).

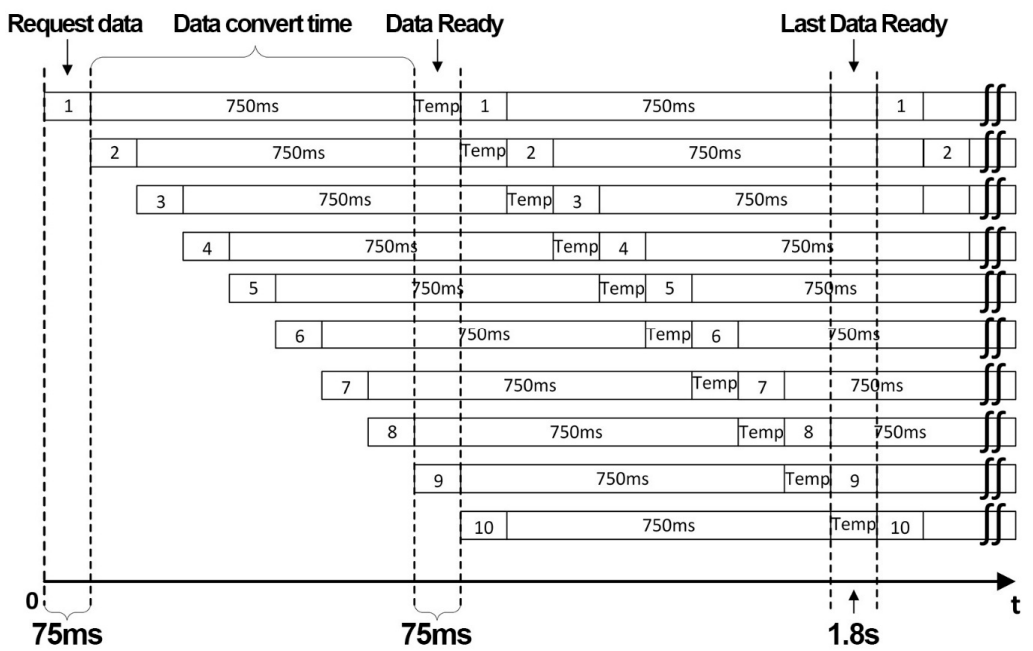

Figure 2. Timing protocol of the signals acquisition system.

A graphical user interface has been also designed for an efficient and easy configuration (Figure 3a) and data management and monitoring (Figure $3 \mathrm{~b}$ ) of the measurement system. Experimental results show valuable performance if compared to those obtained with traditional sensors, i.e., thermocouples and resistance thermometers, both in quasi steady-state conditions and in dynamic conditions. Analog active filters and digital circuits have been used for signal conditioning and data management [7-10]. In addition, a real time clock, the low power DS1307 module provided by Maxim Integrated, has been included in the system and connected through an I2C protocol, in order to track the measurement time. Two experimental tests in quasi steady-state conditions have been carried out, having $200 \mathrm{~min}$ of duration each and with the acquisition time-step of the temperature values equal to $10 \mathrm{~min}$, that is a traditional value in long-term measurements [11]. The acquisition time-step is in agreement with the maximum acquisition rate allowed by the designed system. The obtained results were thus compared, in order to highlight the linearity between the values measured and possible anomalies. 


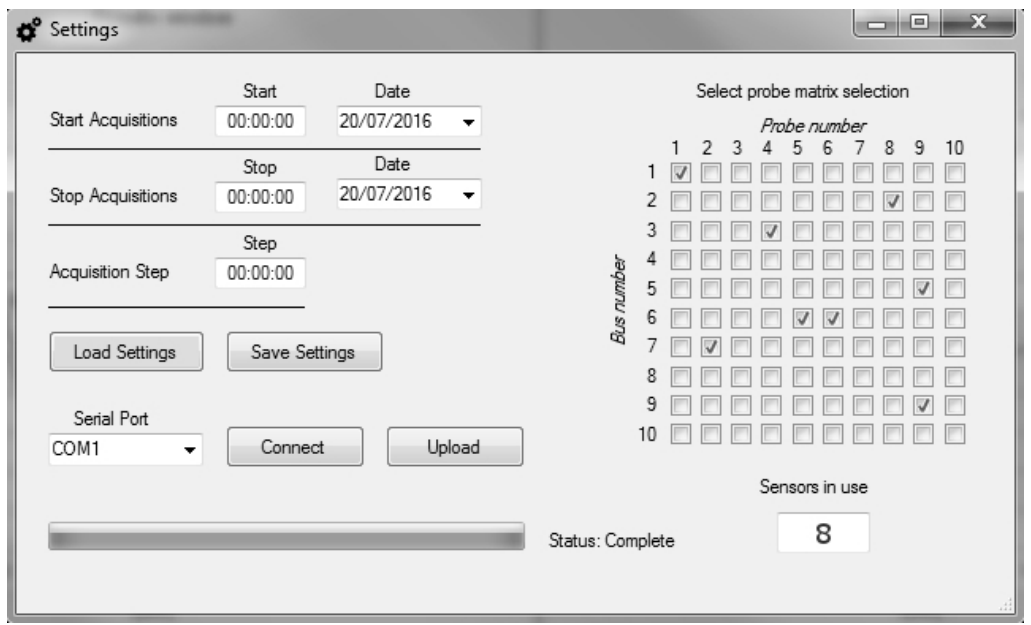

(a)

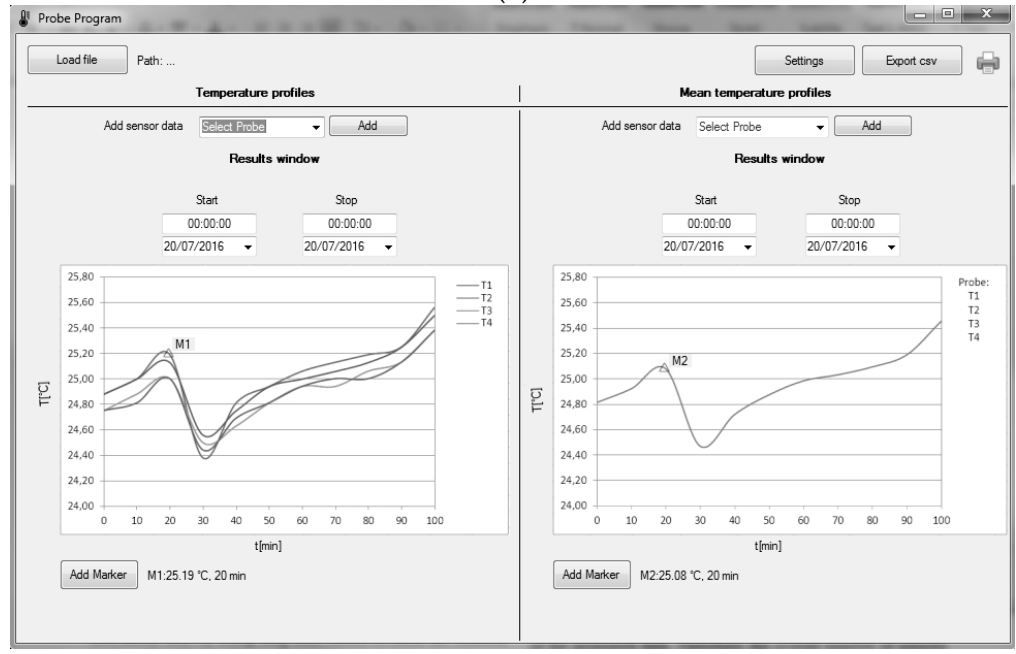

(b)

Figure 3. Designed user interface: (a) Setting utility for user system configuration; (b) Utility for data monitoring.

\section{Conclusions}

In this paper, a novel multi-probes measurement system for on-field analyses of thermal behaviour of structural elements and different building areas is presented. It is important to note that without lack of generality, it is suitable to be employed in each situation that requires a multi-point temperature monitoring, even for long term analyses.

Conflicts of Interest: Authors declare no conflict of interest.

\section{References}

1. Moghavvemi, M.; Ng, K.E.; Soo, C.Y.; Tan, S.Y. A reliable and economically feasible remote sensing system for temperature and relative humidity measurement. Sens. Actuators A Phys. 2005, 117, 181-185. Available online: http://www.sciencedirect.com/science/article/pii/S0924424704004236 (accessed on 28 June 2017).

2. Somov, A.; Baranov, A.; Spirjakin, D. A wireless sensor-actuator system for hazardous gases detection and control. Sens. Actuators A Phys. 2014, 210, 157-164. Available online: http://www.sciencedirect.com/science/article/pii/S0924424714000892 (accessed on 28 June 2017).

3. Kim, Y.; Evans, R.G.; Iversen, W.M. Remote Sensing and Control of an Irrigation System Using a Distributed Wireless Sensor Network. IEEE Trans. Instrum. Meas. 2008, 57, 1379-1387. 
4. Mekki, M.; Abdallah, O.; Amin, M.B.M.; Eltayeb, M.; Abdalfatah, T.; Babiker, A. Greenhouse monitoring and control system based on wireless Sensor Network. In Proceedings of the 2015 International Conference on Computing, Control, Networking, Electronics and Embedded Systems Engineering (ICCNEEE), Khartoum, Sudan, 7-9 September 2015; pp. 384-387.

5. Gad, H.E. Development of a new temperature data acquisition system for solar energy applications. Renew. Energy 2015, 74, 337-343. Available online: http://www.sciencedirect.com/science/article/pii/S0960148114004649 (accessed on 28 June 2017).

6. Ausanio, G.; Barone, A.C.; Hison, C.; Iannotti, V.; Mannara, G.; Lanotte, L. Magnetoelastic sensor application in civil buildings monitoring. Sens. Actuators A Phys. 2005, 123, 290-295. Available online: http://www.sciencedirect.com/science/article/pii/S0924424705001652 (accessed on 28 June 2017).

7. Pantoli, L.; Stornelli, V.; Leuzzi, G. Class AB tunable active inductor. Electron. Lett. 2015, 51, 65-67.

8. Pantoli, L.; Stornelli, V.; Leuzzi, G. Low-noise tunable filter design by means of active components. Electron. Lett. 2016, 52, 86-88.

9. Branchi, P.; Pantoli, L.; Stornelli, V.; Leuzzi, G. RF and microwave high $\mathrm{Q}$ floating active inductor design and implementation. Int. J. Circ. Theor. Appl. 2015, 43, 1095-1104.

10. Pantoli, L.; Stornelli, V.; Leuzzi, G. Tunable active filters for RF and microwave applications. J. Circuits Syst. Comput. 2014, 23, $1450088 . \quad$ Available online: http://www.worldscientific.com/doi/abs/10.1142/S0218126614500881 (accessed on 28 June 2017).

11. Ficco, G.; Iannetta, F.; Ianniello, E.; d'Ambrosio Alfano, F.R.; Dell'Isola, M. U-value in situ measurement for energy diagnosis of existing buildings. Energy Build. 2015, 104, 108-121. Available online: http://www.sciencedirect.com/science/article/pii/S0378778815301158 (accessed on 28 June 2017).

(C) 2017 by the authors. Licensee MDPI, Basel, Switzerland. This article is an open access article distributed under the terms and conditions of the Creative Commons Attribution (CC BY) license (http://creativecommons.org/licenses/by/4.0/). 